\title{
La Mauritanie et la mer : et si le pétrole aidait à mieux gérer l'insécurité écologique?
}

\author{
Géraud Magrin ${ }^{1}$, Geert Van Vliet² ${ }^{2}$ Bopp Van Dessel ${ }^{3}$, Lucien Chabason ${ }^{4}$ \\ 1 Géographe, CIRAD-ES, UMR TETIS / UMR PRODIG, 34398 Montpellier, France \\ 2 Économiste, CIRAD-ES, UMR Art-Dev, 34398 Montpellier, France \\ 3 Écologue, consultant indépendant, 3232 Brielle, Pays-Bas \\ 4 Juriste, conseiller à la direction de l'IDDRI, 75006 Paris, France
}

\begin{abstract}
Natures Sciences Sociétés a souvent évoqué la question des catastrophes et des événements extrêmes (voir les éditoriaux de NSS $[7,3 ; 13,4])$ en souhaitant que les retours d'expérience d'expertises ex post soient valorisés par des expertises ex ante. L'article s'inscrit dans cette perspective. Par l'implication de sciences sociales dans une expertise de risques écologiques, les auteurs montrent que la gouvernance des risques maritimes en Mauritanie, notamment ceux liés à l'extraction pétrolière, ne relève pas seulement de compétences techniques, mais qu'elle renvoie également à des logiques spécifiques de rapports à l'espace. Inversement, par l'importance de la rente qui lui est liée, l'extraction pétrolière joue un rôle de révélateur d'un pouvoir politique national dans ses composantes sociales et leur dynamique. La prise en compte de toutes les activités d'un espace maritime dans leur contexte géopolitique est un exemple de réflexion en vue d'un développement durable pour un pays africain.
\end{abstract}

La Rédaction

\section{Mots-clés :}

multi-usage

de l'espace maritime ; pétrole et gaz offshore ; gouvernabilité ; insécurité écologique ; Mauritanie

\section{Keywords:}

multiuse of maritime space;

offshore oil and gas

exploitation;

governability;

ecological insecurity;

Mauritania
Résumé - Les sociétés qui occupent l'espace de la Mauritanie actuelle ont longtemps tourné le dos à la mer, jusqu'à ce que l'indépendance puis les sécheresses incitent l'État et d'autres acteurs nationaux (commerçants, anciens éleveurs) à s'intéresser aux richesses océanes. En mer comme sur terre, le rapport aux ressources du territoire semble alors celui d'un État rentier, préoccupé de contrôle des flux plus que de gestion. Autour des enjeux de conservation et de pêche, cependant, l'État mauritanien a progressivement renforcé ses capacités. La récente exploitation pétrolière offshore, au-delà de ses enjeux spécifiques, a souligné l'importance d'améliorer la gouvernance maritime d'ensemble, qui inclut les relations entre conservation, pêche, transport et activités pétrolières et gazières. Elle pose en des termes nouveaux la question de l'appropriation et de la gestion de l'espace et de ses ressources par un État dépendant, dont le territoire suscite l'intérêt de nombreux acteurs internationaux.

\footnotetext{
Abstract - Mauritania and its sea: could offshore oil exploitation help better manage ecological insecurity? Over centuries Mauritanian societies have turned their backs to the sea until independence and, later, drought created incentives for the State and national actors to take an interest in sea resources. Whether at sea or on the mainland the relationship to the territory's resources appeared to be entrusted to the same rentier State who was more interested in exercising control on flows rather than in management, production or transformation. However, regarding the fisheries industry and the issue of its preservation the Mauritanian state has progressively increased its intervention capacities. The extraordinary ecological and economic value of Mauritanian waters - with the "banc d'Arguin" as their most famous ecosystem certainly played a role in this evolution. The recent exploitation of offshore oil resources has reinforced the need to increase the overall capacity to regulate all aspects of the maritime area (conservation, fisheries, transport and oil and gas). The emergence of oil and gas activities re-emphasises the difficulties faced by a dependent State when trying to appropriate and manage a territory whose resources attract the attention of powerful international stakeholders.
} 


\section{Introduction}

L'histoire de la gestion des eaux mauritaniennes $^{1}$ depuis l'indépendance, en 1960, est celle d'une interrelation asymétrique entre les autorités nationales et des acteurs extérieurs. Longtemps, la Mauritanie ne contrôla guère son espace maritime. Progressivement, des régulations ont été mises en place dans le secteur de la pêche et de l'environnement, avec des appuis extérieurs plus ou moins désintéressés. Mais l'histoire postcoloniale a aussi fait de l'État mauritanien un État rentier, à l'image des autres pays subsahariens (Magrin, 2010), c'est-à-dire un État extraverti et dépendant de l'aide internationale, aux fonctions de contrôle et de planification territoriale atrophiées. L'apparition du pétrole a complexifié ce système rentier - qui repose désormais sur quatre piliers, à savoir la mine $^{2}$, la pêche, le pétrole et l'aide internationale ${ }^{3}$ tout en rendant plus évidents les besoins de régulation du multi-usage de l'espace océanique, en particulier du fait des risques écologiques de l'offshore.

Notre réflexion s'insère dans le cadre des travaux sur la «malédiction des ressources naturelles » dans les pays les moins avancés, qui montrent que les pays aux institutions faibles riches en matières premières connaissent un certain nombre de difficultés communes. Celles-ci concernent notamment la croissance économique, la gouvernance (régimes politiques, corruption, conflits) [cf.par exemple Rosser, 2006; Humphreys et al., 2007; Soares de Oliveira, 2007] et l'environnement. Nous proposons de décliner cette réflexion à propos des relations entre la Mauritanie, son espace maritime, ses ressources et les acteurs qui y interviennent. Ce texte se donne comme objectif de montrer comment cet État gère la complexité croissante des activités maritimes et les risques qui y sont associés. L'émergence d'une conscience de la fragilité de

\footnotetext{
${ }^{1}$ Cet article mobilise notamment les acquis des auteurs au sein du Panel scientifique indépendant sur les activités pétrolières et gazières en Mauritanie. Si le contenu du texte n'engage que les auteurs, ceux-ci tiennent à remercier pour les informations partagées les responsables du programme Global et du programme Entreprises et biodiversité de l'UICN (Gland), ainsi que, à Nouakchott, les participants de la Plateforme de dialogue sur les activités pétrolières, MM. Olivier Ruë et Antonio Araujo, experts au Parc national du banc d'Arguin (PNBA), les membres de l'équipe UICN (MM. Mohamed Lemine Ould Baba, Abderrahmane Limane, Mathieu Ducrocq, Mme Aminetou Mint Kebd) et M. Isselmou Abdelkader.

2 L'essentiel de la rente minière vient de la mine de fer de Zouerat, exploitée depuis la fin des années 1950, nationalisée en 1974 et gérée depuis par la SNIM (Société nationale d'industrie minière) [Bonte, 2001]. S'y ajoutent, de manière secondaire et intermittente, les revenus d'autres mines (cuivre et or d'Akjoujt et, plus récemment, de Taziast).

3 À ces flux légaux s'additionnent, pour certains acteurs opérant aux marges du système politico-économique national, des revenus captés sur les trafics sahariens (contrebande, drogue, etc.).
}

la ressource peut-elle être à l'origine d'une bifurcation de la trajectoire de l'État hors des sentiers (mal) fréquentés des victimes de cette «malédiction» (Magrin et Van Vliet, 2009)?

Notre réflexion s'inscrit, en outre, dans un contexte marqué par l'incertitude politique et pétrolière. Le président Maouiyya Ould Taya a été renversé par un coup d'État en août 2005. Un régime de transition a conduit à l'élection, en avril 2007, d'un nouveau président, Sidy Ould Cheikh Abdallahi. Celui-ci a été à son tour renversé par un putsch en août 2008, déclenchant une crise politique qui semble se résoudre par l'élection, le 18 juillet 2009, du général responsable de ce dernier coup d'État, Mohamed Ould Abdel Aziz. Ces événements n'ont pas été sans effet sur les relations entre l'État et le pétrole (Augé, 2007). L'Australien Woodside, qui a découvert et commencé d'exploiter le gisement offshore de Chinguetti en février 2006, a laissé sa place au Malaisien Petronas en janvier 2008, sans que cela réponde aux questions qui planent sur l'avenir pétrolier du pays ${ }^{4}$.

Nous chercherons donc à vérifier les hypothèses suivantes :

- dans un vaste État sahélo-saharien comme la Mauritanie, faisant partie des pays les moins avancés, le pouvoir repose sur un système rentier organisé autour du contrôle des circulations et des relations extérieures (flux de produits, de ressources) plutôt que sur le contrôle, l'administration et la gestion du territoire. De ce point de vue, la mer vaut la terre : Sahara et océan participent d'un même rapport à l'espace de l'État et de la société; toute nouvelle rente, quelle que soit son origine, peut être absorbée sans modifier le système ;

- la perspective de l'exploitation pétrolière a eu des effets inattendus : l'anticipation de ses impacts a cristallisé des débats houleux, mais la question pétrolière a aussi révélé la complexité de la gestion d'usages superposés de l'espace maritime et le déficit de moyens techniques, scientifiques et financiers disponibles pour y répondre. La prise de conscience des risques environnementaux ${ }^{5}$ de l'exploitation pétrolière met l'État devant des choix qui dépassent cette activité, et permet de mieux appréhender l'insécurité écologique qui caractérise les milieux marins et littoraux.

\footnotetext{
${ }^{4}$ Ce gisement est jusqu'à présent décevant : on prévoyait au moins 60000 barils par jour $(\mathrm{b} / \mathrm{j})$, mais la production ne dépassait pas $10000 \mathrm{~b} / \mathrm{j}$ fin 2010. L'avenir pétrolier dépend donc des prospections en cours.

5 C'est à la suite du différend qui éclata entre la Mauritanie et Woodside, en 2006, que le gouvernement demanda à l'UICN de réunir un panel scientifique indépendant pour éclairer les décisions susceptibles de prévenir les risques associés à l'activité pétrolière.
} 
Avant la colonisation

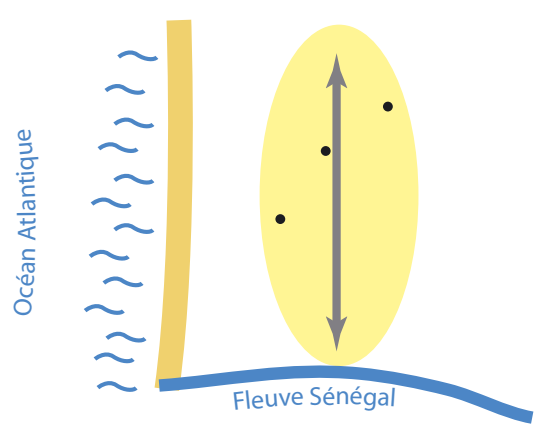

Aujourd'hui

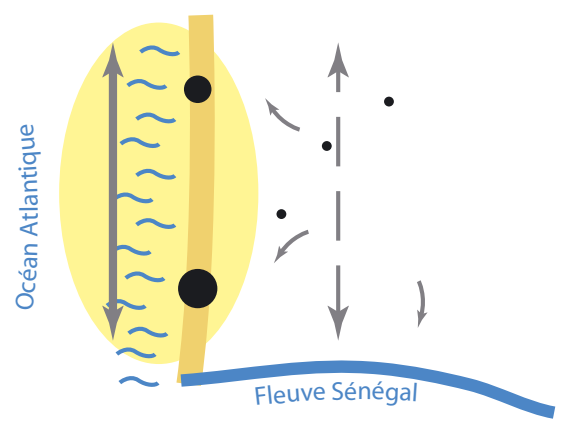

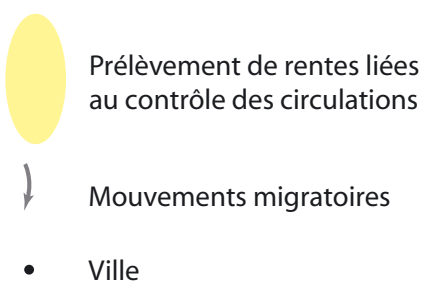

Fig. 1. Littoralisation des circulations et des rentes territoriales en Mauritanie (schéma réalisé avec l'aide de D. Gautier).

Nous rappellerons d'abord les caractéristiques des rapports entre pouvoir et espace en Mauritanie, basés sur le contrôle des flux plutôt que sur celui des territoires. Puis nous décrirons la montée en puissance du secteur maritime (pêche, transport) et présenterons les enjeux de régulation liés à l'irruption du pétrole. Nous analyserons enfin les modifications en termes de gouvernabilité ${ }^{6}$ de l'espace maritime issues de ce nouveau contexte.

\section{Société et espace : un continuum du désert à l'océan}

Il s'agit ici de s'interroger sur l'appropriation de l'espace océanique par la Mauritanie. Éloignées de la mer, les sociétés rassemblées dans ce qui constitue aujourd'hui le territoire mauritanien (Marchesin, 1992 ; Antil, 1999) s'en sont rapprochées à la faveur des mutations qui jalonnent un demi-siècle d'indépendance. De l'éleveur nomade au

\footnotetext{
${ }^{6}$ La gouvernabilité est relative, elle n'existe pas en soi. Tout décideur, où qu'il soit, dispose de marges de manœuvre plus ou moins étroites, qui sont la manifestation de la « gouvernabilité du système » (Van Vliet, 1997). Ces marges semblent encore plus limitées pour les États du Sud (en particulier ceux des pays les moins avancés). Selon Matus (1987), la gouvernabilité dépend de trois facteurs liés : la nature (simple ou complexe) des problèmes à traiter ; la qualité du projet présenté par l'acteur (qui dépend de sa légitimité politique et sociale, de sa pertinence par rapport au problème et de la base de ressources dont il dispose); la capacité de gouverner, c'est-à-dire de mobiliser et d'utiliser les ressources (temps, connaissances, espace, ressources naturelles, capacité organisationnelle, capital politique, ressources financières).
}

citadin pêcheur, le changement semble saisissant. Il ne l'est pas tant si on se réfère aux rapports à l'espace mis en œuvre : au Sahara comme sur mer, le pouvoir tient au contrôle des circulations plus qu'à la maîtrise du territoire (Walther et Retaillé, 2008).

\section{La mer confins}

En dépit d'une ouverture littorale importante - près de $700 \mathrm{~km}$ de côtes -, les sociétés qui peuplent l'actuelle Mauritanie ont longtemps négligé la mer. Les Maures qui nomadisaient entre le fleuve Sénégal et le Sahara étaient des éleveurs sahélo-sahariens et des commerçants; les Toucouleurs de la rive droite de la vallée du fleuve Sénégal pratiquaient l'agriculture, l'élevage ou la pêche fluviale. Pour eux, le littoral était répulsif en raison de l'absence d'eau douce accessible et des relations difficiles avec l'arrière-pays qu'opposaient de hautes dunes côtières (Le Cœur, 1994). La traite associée à la fréquentation européenne du littoral, à partir du XV $\mathrm{XV}^{\mathrm{e}}$ siècle, rendit en outre ces zones dangereuses. Aussi restèrent-elles longtemps quasiment désertes. Les rares populations littorales vivaient à la périphérie du territoire actuel - au Nord, les Imraguen, tributaires de groupes maures (Ould Cheikh, 2002 ; Boulay, 2008) ; au Sud, des populations wolof proches de la frontière sénégalaise.

De même, l'organisation urbaine de la Mauritanie a évité la mer jusqu'au début des années 1960. Une ancienne vie urbaine fut associée, dans l'intérieur, au commerce transsaharien du sel et de la gomme - sans toutefois susciter l'essor de villes importantes (Fig. 1). À l'époque coloniale, on se contenta d'administrer le 
territoire depuis Saint-Louis. Un port fut tout de même créé : Port Étienne (actuel Nouadhibou), né du poisson, d'un bon site portuaire sur une côte qui en comptait peu, puis de l'exportation du fer (Toupet, 1968; Bonte, 2001). Mais sa position périphérique et l'absence de communications routières avec le reste du territoire l'enfermaient dans un arrière-pays très exigu.

Malgré les évolutions ultérieures, l'océan est resté discret dans les représentations nationales. Un ouvrage sur les 50 ans de Nouakchott (Ministère de la Culture, de la Jeunesse et des Sports, 2006) met en scène une représentation discutable d'une Mauritanie essentiellement bédouine et beydane ${ }^{7}$, nomade jusque dans les éléments modernes de la ville, dont les composantes noires et négro-africaines sont quasiment absentes (Choplin, 2006). On pourrait, de même, constater la discrétion de l'océan. Dans cet ouvrage très bien illustré ne figurent qu'une seule photographie du port - moderne - de l'Amitié et une image d'une bassine de poissons. Ni les échanges maritimes ni la pêche ou les pêcheurs ne sont valorisés dans une iconographie qui fait une large place au contraste de la dune, du chameau et de l'urbanisme contemporain. De même, on peut s'interroger sur le sens du nom - Chinguetti - donné au premier gisement de pétrole exploité en Mauritanie, situé en mer à $80 \mathrm{~km}$ au sud-ouest de Nouakchott. Il signale, à n'en pas douter, le succès de mémoire d'une ancienne histoire urbaine saharienne, inscrite dans le registre idéologique (ibid.) de la «maurité ». Mais il manifeste ainsi avec force, par la charge symbolique dont il est investi, l'intégration de l'espace océanique au territoire national.

\section{Ruée vers l'eau}

La Mauritanie est pourtant venue au rivage. L'indépendance s'inaugure par l'érection ex nihilo d'une capitale, Nouakchott, dont la situation est influencée par la position méridienne centrale au sein du territoire et l'ouverture maritime. Si l'océan valorise la situation de la ville, le site s'en tient à l'écart. Ni front de mer ni, encore moins, vieux port : le centre est situé à une dizaine de kilomètres de la côte ${ }^{8}$.

Nouakchott connaît une croissance extrêmement rapide, au point de passer de quelques milliers d'habitants en 1960 à près d'un million en 2008 , concentrant près du tiers de la population nationale. Aux facteurs habituels de la croissance urbaine dans les pays du Sud - rôle urbanisant de l'État, interface avec l'économie mondiale,

\footnotetext{
${ }^{7}$ Les lectures habituelles de la société mauritanienne distinguent les Beydan (Maures blancs), les Haratin (Maures noirs) et les Négro-Africains (populations de diverses ethnies vivant le long de la vallée du Sénégal).

8 Nouakchott fut à l'origine un poste militaire créé en 1903 autour d'un point d'eau connu des nomades des environs. Il servit de relais sur la piste impériale ${ }^{\circ} 1$ Saint-Louis - Atar.
}

etc. - se sont ici ajoutés les effets brutaux des sécheresses des années 1970-1980. En détruisant les ressources pastorales et les troupeaux, ces décennies sèches ont provoqué au Sahel une brutale accélération de l'urbanisation, qui, en Mauritanie, s'est concentrée dans deux villes littorales : Nouadhibou et, bien davantage, Nouakchott.

C'est dans ce contexte de crise climatique, politique (la guerre au Sahara occidental affecte le Nord du pays) et économique (les cours du fer diminuent sur les marchés mondiaux) que l'État et la société mauritanienne vont s'intéresser aux ressources de la mer. La richesse halieutique des eaux littorales des côtes du Nord-Ouest de l'Afrique est favorisée par la dérive canarienne et un puissant phénomène d'upwelling - qui désigne la remontée d'eaux profondes riches en nutriments. Elle est renforcée par la présence au nord du pays du golfe d'Arguin, écosystème original à l'extraordinaire productivité biologique. Cet environnement est donc extrêmement précieux, tant du point de vue économique que de celui des enjeux écologiques de conservation.

La pêche s'est développée tout au long du XXe siècle, au gré des progrès techniques permettant $\mathrm{d}^{\prime}$ attraper, de conserver et de transporter le poisson. À partir des années 1960, la pêche artisanale connaît un développement important. Mareyeurs et usines mobilisent des milliers de pêcheurs de la sous-région et font travailler un grand nombre de petits commerçants, transformateurs et transporteurs. L'État lance en 1977 une politique de pêche nationale ambitieuse. En 1979, il achète d'occasion une centaine de navires de pêche industrielle, avec l'aide de pays arabes recyclant des pétrodollars (Le Cœur, 1994) - première rencontre, indirecte, de la pêche et du pétrole en Mauritanie -, et renforce son contrôle des eaux et de l'ensemble de la filière. Cette flotte sera bientôt en faillite, mais elle marque une première tentative significative d'amélioration de l'emprise nationale sur les ressources halieutiques. Les activités liées à la mer (pêche, mais aussi transport et commerce maritime) acquièrent ainsi une place croissante dans le système politico-économique extraverti qui se consolide parallèlement à l'État mauritanien (Choplin et Lombard, 2009).

La pêche pèse à présent un poids très conséquent dans l'économie nationale (Marfaing, 2005). Elle fournit environ $25 \%$ des revenus de l'État et du PIB, $50 \%$ des recettes d'exportation, l'essentiel (70\%) des 100000 tonnes de production annuelle de poisson étant exporté. Les 450 navires de la flotte industrielle se partagent entre Nouadhibou et Nouakchott. Avec les 3000 pirogues de la pêche artisanale, ils représentent près de 40000 emplois directs et de nombreux emplois indirects ${ }^{9}$. Mais la pêche industrielle repose en grande partie sur des flottes

\footnotetext{
9 Il est difficile d'apprécier les parts respectives des pêches artisanale et industrielle dans la production, dans la mesure où les pirogues artisanales livrent une part conséquente de leur pêche aux navires industriels.
} 
étrangères, qui travaillent dans le cadre d'accords avec le gouvernement mauritanien. Les chalutiers opèrent à une grande variété de profondeur (jusqu'à 800 m et plus) ; ils sont parmi les plus grands navires de ce type en service actuellement dans le monde.

Mer et terre renvoient finalement à des formes de rapport à l'espace comparables (Fig. 1). Au-delà des oppositions entre genres de vie (nomades/sédentaires), au Sahara et au Sahel, le pouvoir réside dans la maîtrise de la mobilité, des circulations, des flux - et des lieux fixes qui les sous-tendent (Retaillé, 2006) - plus que dans le contrôle de l'étendue territoriale elle-même. La construction d'un État, engagée au moment de l'indépendance, s'effectue donc dans des conditions paradoxales : l'État cherche à s'approprier son territoire et des populations dont les paradigmes demeurent la mobilité et la plasticité des découpages (Lechartier, 2005). Sahara et océan lui opposent des enjeux de contrôle territorial analogues - les moyens techniques, financiers et humains nécessaires au contrôle de la distance se révélant très difficiles à réunir pour un État à faible base fiscale. Ces difficultés sont ensuite d'autant plus grandes que ce tournant vers la mer s'est effectué dans le contexte de crise politicoéconomique qui a affecté toute l'Afrique à partir des années 1980. Comme ailleurs, l'ajustement structurel a fragilisé les fonctions de légitimation de l'État qui avaient commencé à être mises en place au lendemain des indépendances, au profit d'un approfondissement des stratégies rentières et clientélistes d'élites amincies (Bayart, 1999). L'affaiblissement de l'État mauritanien consécutif à la sécheresse, à la crise du Sahara occidental et aux difficultés économiques s'est accompagné d'un renforcement des inégalités sociales et d'une accumulation des richesses au sein de certaines tribus ${ }^{10}$. Le boom de la pêche a ainsi permis un enrichissement rapide des proches $\mathrm{du}$ pouvoir - à travers, par exemple, la vente de licences de pêche (Marchesin, 1992). Mais, de même que l'appartenance tribale ne constitue qu'un élément explicatif parmi d'autres de la réussite politique et économique (Blundo, 2007), on ne peut en déduire un positionnement simple dans le secteur halieutique.

\footnotetext{
${ }^{10}$ Un petit nombre de tribus concentre l'essentiel du pouvoir et des richesses, dessinant un "triangle de la fortune » entre l'Adrar au nord, Tidjikja au centre-est et Akjoujt à l'ouest (Blundo, 2007) : les Awlad Busba et les Idawali de l'Adrar (Chinguetti) et du Tagant (Tidjikja) sont à l'origine des premières fortunes sahariennes (Bonte, 2000). Les Smassid d'Atar ont connu une ascension remarquable après la Deuxième Guerre mondiale, stimulée ensuite par leurs liens avec le régime du président Ould Taya, qui appartient à ce groupe et a intensifié les relations entre pouvoir politique, acteurs économiques et militaires. Depuis quelques années, les Awlad Busba d'Akjoujt ont bénéficié de la présence à la tête de l'État de deux des leurs : le leader de la transition Mohamed Vall, puis l'actuel président Abdel Aziz (Choplin et Lombard, 2009).
}

Entre contrôle continental et maritime, une différence de taille tient à l'inégale valeur des deux étendues dans le contexte de la mondialisation : les richesses de la mer (poisson, pétrole, voire biodiversité) sont convoitées par des acteurs extérieurs multiples, ce qui y complique les enjeux de contrôle territorial.

\section{Le pétrole comme révélateur des fragilités océanes}

Il existe une abondante littérature, scientifique ou de vulgarisation, sur la richesse et la fragilité des écosystèmes marins et littoraux de la Mauritanie ${ }^{11}$. Au début des années 2000, la découverte du pétrole au large des côtes mauritaniennes révèle des contradictions latentes entre les différents usages de l'espace maritime et la complexité des enjeux environnementaux associés.

\section{Les premiers conflits d'usage maritimes}

C'est à propos de la pêche que se sont noués les premiers conflits d'usage de l'espace maritime en Mauritanie. Ils expriment la concurrence pour l'accès aux zones de pêche entre groupes, nationalités ou types d'activité. Les tensions principales émaillent depuis longtemps les relations entre pêches artisanale et industrielle. Depuis les accords de pêche de 1995, les chalutiers sont censés épargner la zone littorale sur une bande de 3 à 12 miles (Fig. 2). Dans la réalité, ils transgressent fréquemment cette interdiction. Pêche artisanale et pêche industrielle se rejettent constamment la responsabilité de la diminution de la ressource.

Les découpages de l'espace maritime résument bien l'évolution et la complexification des enjeux de gouvernance de la mer mauritanienne (Le Cœur, 1994), mais aussi la rapidité de la prise de conscience par l'État de ces enjeux. En 1967, les eaux territoriales sont délimitées à 12 miles, puis étendues à 70 miles en 1978, en même temps qu'est dessinée une zone économique exclusive (ZEE) de 200 miles (Fig. 2). La Mauritanie est ainsi un des derniers États à s'être approprié juridiquement son espace maritime. Plusieurs générations de découpages de l'espace de pêche se succèdent ensuite. Actuellement, on distingue une zone Nord, une zone Centre et une zone Sud, au sein de laquelle un zonage plus fin par pêcheries est utilisé, défini en concertation avec les pêcheurs. La Mauritanie apparaît en avance sur les pays voisins dans le domaine de la gestion de la pêche.

\footnotetext{
11 Voir les études réalisées par le PNBA et la Fondation internationale du banc d'Arguin (FIBA) sur le banc d'Arguin, dont $l^{\prime}$ « Étude d'impact environnemental » de Woodside (2003 et 2005) synthétise une bonne part; et Campredon (2000) pour une version grand public.
} 


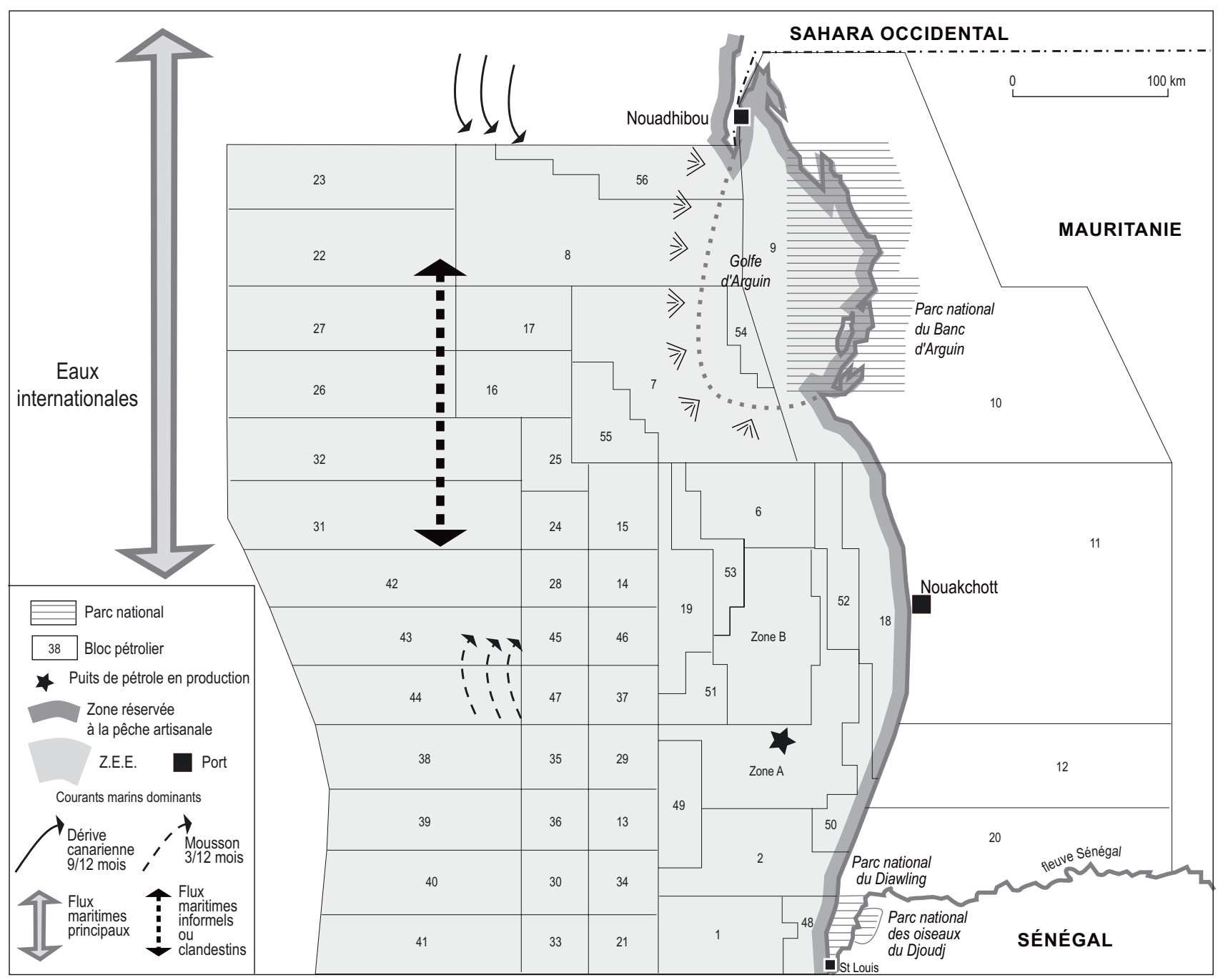

(c) Conception : G. Magrin. Réalisation : C. Carrié

Fig. 2. Enjeux écologiques des usages de l'espace maritime mauritanien.

Le découpage des concessions pétrolières s'est surimposé à cette fine trame de maillage de l'espace halieutique. Les activités industrielles y sont pourtant encore limitées, mais elles suffisent à soulever des questions importantes sur les enjeux de cohabitation entre une activité industrielle par nature polluante et des écosystèmes fragiles économiquement vitaux pour un pays pauvre.

\section{Les risques pétroliers éclairent ceux des autres usages marins}

La fragilité écologique des eaux et rivages de la Mauritanie tient à la présence conjointe d'écosystèmes sensibles et d'activités humaines de plus en plus intensives. L'exploitation pétrolière récente est lourde de menaces pour les écosystèmes marins ; elle a en outre révélé les risques latents liés au transport maritime international le long des côtes.
Dans un contexte de hausse mondiale des prix du pétrole, la découverte en 2001, par l'entreprise australienne Woodside, du gisement offshore de Chinguetti a déclenché une véritable euphorie pétrolière, assortie d'une intensification de la prospection. L'essentiel du domaine maritime national, qu'il soit côtier ou non, fut découpé en blocs (Fig. 2) [Augé, 2007]. Durant la période précédant le début de l'exploitation, puis au début de celle-ci, des débats très vifs ont opposé des organisations de la société civile internationale, le gouvernement mauritanien et l'entreprise pétrolière. Ceux-ci tournaient pour l'essentiel autour de la gouvernance des revenus pétroliers (Oilwatch, 2005) et des impacts sur l'environnement marin des activités offshore (Kloff et Van Spanje, 2004; Kloff, et Wicks, 2005 ; Goodland, 2006).

Les risques liés aux activités pétrolières et gazières dépendent de deux facteurs : la nature des dangers, selon le type d'activité, et la sensibilité de l'environnement. Si le danger ou la sensibilité n'existe pas, il n'y a pas 
d'impact possible. Trois risques environnementaux principaux sont ici identifiés. Les mesures de prévention ou de réponse qu'ils commandent (Van Vliet et al., 2009) prennent tous en compte la nature unique et fragile de l'environnement des côtes ouest-africaines, leur importance socioéconomique majeure pour le domaine de la pêche - essentielle pour l'emploi local et le revenu national -, de même que la capacité actuelle de l'État à y faire face.

Le premier risque est lié au déversement accidentel ou à des fuites de pétrole. Il est majeur dans les zones riches en poissons ou importantes pour leur reproduction - comme le banc d'Arguin. Au-delà de la prévention, la réaction à des accidents pose problème. Les opérateurs du champ de Chinguetti (Petronas et ses sous-traitants) ont des capacités de réponse d'urgence adaptées à de petites fuites. Mais la Mauritanie n'est pas préparée à des situations d'urgence à une échelle nationale ou sousrégionale et ne dispose ni des moyens techniques ni des mécanismes de coordination pour y répondre. Le second risque concerne l'utilisation des ondes sismiques sonores envoyées depuis la surface (à l'aide d'un canon à air ou à eau), une technique connue pour ses effets négatifs sur les mammifères marins et les larves de poisson (Semelin, 2005). Le troisième risque renvoie à la gestion des déchets de l'industrie pétrolière.

Enfin, ces risques dépendent de l'ampleur des activités. La production de Chinguetti n'atteint que $15 \%$ du volume prévu, et personne ne connaît l'issue des prospections en cours. L'avenir est donc incertain : boom ou fin de l'ère pétrolière peuvent survenir demain, avec des conséquences très différentes sur les risques environnementaux afférents. Dans les débats sur la compatibilité entre exploitation pétrolière et préservation de l'environnement marin, le banc d'Arguin occupe une place emblématique. De nombreuses organisations de coopération bilatérale et de la société civile internationale ont permis la protection d'une partie du golfe d'Arguin par la création, en 1976, du Parc national du banc d'Arguin (PNBA), classé au patrimoine naturel mondial de l'Unesco en 1989 (Campredon, 2000). En mars 2008, un projet d'arrêté a été proposé par le ministère du Pétrole pour modifier le découpage de certains blocs pétroliers, notamment autour du banc d'Arguin. Celui-ci occupe une partie importante des blocs 9 et 10 (Fig. 2), qui, de ce fait, n'étaient pas attribués. Il s'agissait donc d'ouvrir aux activités pétrolières (exploration, puis éventuellement production) les parties de ces deux blocs extérieures au PNBA. L'administration du parc, les ONG environnementales (UICN, FIBA), puis le Panel scientifique indépendant sur les activités pétrolières et gazières ont montré les dangers d'une telle modification du statu quo ante (Ruë, 2005; Van Vliet et al., 2009). Le contexte d'instabilité politique qui caractérise la Mauritanie depuis 2005 a empêché l'État, jusqu'à présent, de trancher entre un principe de précaution environnementale appliqué à une zone vitale et l'espoir d'augmenter la rente pétrolière. L'arrêté est (provisoirement) enterré. Les arbitrages à venir sur le sujet seront un bon témoin des rapports de force à l'œuvre entre lobbys pétrolier et environnementaliste.

En outre, l'espace maritime mauritanien est parcouru du nord au sud par le rail de navigation ouest-africain (Fig. 2). Des milliers de navires croisent annuellement au large de ces côtes. Les problèmes liés à ce trafic intense ont été identifiés depuis longtemps (gestion des déchets des navires, dégazages, accidents, risques de marée noire), mais les capacités de régulation et d'intervention des autorités mauritaniennes n'ont guère évolué. Les débats soulevés par les perspectives pétrolières au début des années 2000 ont exhumé ces enjeux, en rappelant l'acuité des risques environnementaux liés à la sensibilité du banc d'Arguin. En l'état actuel des choses, le risque pour le golfe d'Arguin d'un accident de navigation affectant, au nord des eaux mauritaniennes, un navire transportant des hydrocarbures semble au moins aussi grand que celui représenté par une marée noire issue du champ de Chinguetti.

Or, malgré les avertissements de nombreux experts, le gouvernement mauritanien n'a pas ouvert de négociations avec l'Organisation maritime internationale (OMI) sur la modification de l'axe de navigation. Le tracé actuel, qui repose plus sur des habitudes de navigation que sur l'évaluation des risques, ne prend en compte ni les impératifs environnementaux (écosystèmes marins et côtiers les plus fragiles) ni les contraintes économiques (zones de pêche, activités pétrolières). Cette inaction doit-elle être imputée à la faiblesse technique de l'administration nationale ou à sa faible perception de l'intérêt d'une lourde procédure en réponse à des problèmes trop virtuels ? Probablement un peu aux deux.

\section{Gouverner la mer sous contraintes sévères}

La complexité des enjeux de gouvernance maritime en Mauritanie tient au multi-usage croissant de cet espace et de ses ressources, au poids de nombreux acteurs extérieurs et aux capacités encore limitées de coordination de l'État.

\section{Un espace maritime sous influences extérieures}

Depuis 1960, le jeune État mauritanien semble renforcer progressivement sa souveraineté sur son espace maritime et ses ressources. Mais il a dû composer avec des tiers originaires de pays variés (France, Espagne et autres États de l'Union européenne, Japon, pays d'Europe de l'Est, Malaisie, Sénégal, etc.), qui avaient, pour 
la plupart, tissé des liens avec l'océan mauritanien et ses ressources bien avant l'indépendance. Cette influence internationale multiforme s'exerce dans les domaines de la pêche, de la protection des écosystèmes marins et côtiers, des transports ou des hydrocarbures. Aides et intérêts économiques coïncident souvent. Les enjeux de gestion de l'espace maritime contribuent, de manière plus ou moins directe, à justifier l'action d'un certain nombre de bailleurs, intéressés à l'exploitation (pêche pour la France, l'Espagne, l'Union européenne, le Japon, etc. ; pétrole), la conservation (pays de l'OCDE surtout) ou le contrôle de cet espace (pays européens pour le contrôle des flux migratoires et des trafics).

La présence étrangère dans le domaine de la pêche a une longue histoire (Le Cœur, 1994). La pêche industrielle canarienne s'est implantée dans l'espace maritime mauritanien au début du $X^{\mathrm{e}}$ siècle. Une politique active de licences de pêche a été mise en place dès 1967; les flottes des pays de l'Est sont venues s'ajouter aux bateaux d'Espagne, de Cuba, d'Afrique du Sud, du Japon, d'Israël ou d'Égypte. En 1983, la Mauritanie et le Sénégal signent une convention qui autorise l'accès à la mer mauritanienne aux pêcheurs sénégalais. Les premiers accords de pêche avec la CEE sont signés en 1987. En 1994, la flotte européenne opérant dans les eaux mauritaniennes comptait plus de cent navires (un tiers de la flotte étrangère). Enfin, il faut signaler que la gestion des pêches est partiellement internationalisée, puisque, depuis 1985, la commission régionale des Pêches essaie de formuler des solutions pour limiter la surpêche (réglementations sur les rythmes de pêche et les repos biologiques, les engins, etc.).

Le bilan à long terme de l'exploitation des ressources marines par des compagnies étrangères est ambigu. Alors que les bénéfices du secteur de la pêche semblent plus élevés à Las Palmas qu'à Nouadhibou, et que seules certaines espèces parmi les moins rémunératrices ont été jusqu'alors exploitées par les acteurs mauritaniens, l'argent obtenu grâce aux licences n'a été que marginalement réinvesti dans une capacité de pêche et de gestion de la pêche nationale, comme le signalait déjà Le Cœur (1994). Les innombrables projets de coopération en faveur de la pêche (CEE puis UE, Japon, FAO, aide bilatérale italienne, danoise, etc.) qui ont accompagné les négociations successives des accords se sont traduits par la construction d'infrastructures, notamment dirigées vers la pêche artisanale, sans toutefois susciter de dynamiques autonomes. Alors que l'Union européenne déploie des financements importants pour des projets en faveur du développement durable, le système des accords de pêche dont elle est l'acteur central contribue à la dégradation des ressources halieutiques nationales (Ould Ahmed Salem, 2008). À tel point que, récemment, on a pu envisager, au sein de Greenpeace, que les pratiques de pêche puissent avoir finalement des impacts écologiques infiniment supérieurs à ceux des activités pétrolières actuelles ${ }^{12}$.

Enfin, les écosystèmes du banc d'Arguin ont fait l'objet de coopérations extérieures considérables avant même la création du PNBA. Ces appuis ont permis de contribuer à la protection des ressources naturelles, sans éviter des critiques liées à la dépendance associée à l’« aide verte $^{13}$ ». Allouée sous forme de nombreux projets, celleci s'est traduite par la construction d'infrastructures (radars de surveillance, station scientifique, eau, accueil de visiteurs, magasins communautaires), la réalisation de recherches scientifiques et de formations et l'appui budgétaire au fonctionnement du parc. Bien que l'État mauritanien ait peu à peu augmenté sa contribution financière ${ }^{14}$, il reste marqué par une forte dépendance visà-vis de l'aide internationale et par une faible autonomie technique nationale (Van Vliet, 2002).

Enfin, l'histoire pétrolière de la Mauritanie reste à écrire. Une grande variété de firmes étrangères ${ }^{15}$ mènent des activités de prospection, dans un contexte marqué par l'incertitude, la faiblesse de la société civile nationale et l'impréparation apparente de l'État. Les rapports entre ce dernier et les firmes pétrolières témoignent-ils de l'asymétrie habituelle des débuts de cycle pétrolier en faveur des entreprises étrangères ou, au contraire, l'État utilise-t-il dans les négociations pétrolières l'expérience accumulée autour du fer et de la pêche?

\section{L'État empêtré dans ses propres filets}

Les capacités maritimes de l'État mauritanien traduisent la montée en puissance de l'intérêt pour les ressources de l'océan. Les secteurs de la pêche et de la marine marchande se sont renforcés avec le temps, même si

12 Communication de Sandra Kloff aux auteurs, juin 2009.

13 L'expression désigne l'aide associée à la protection de l'environnement. Elle repose sur l'idée que les pays du Nord doivent aider ceux du Sud à protéger des ressources naturelles qui sont parfois affectées, directement ou indirectement, par l'action d'acteurs du Nord, ces ressources étant ainsi perçues comme des biens publics mondiaux. Cette forme d'aide est parfois critiquée pour sa dimension exogène, la faible appropriation des enjeux et dispositifs par les acteurs locaux et sa contribution au processus de fragmentation territoriale (à travers la création d'aires protégées plus ou moins extraterritoriales) qui rend le gouvernement des territoires nationaux encore plus difficile (Ferguson, 2006).

14 Depuis 2006, une part du flux financier issu des accords de pêche avec l'Union européenne est affectée au développement durable, en particulier à l'Institut mauritanien de recherches océanographiques et des pêches (IMROP) et au PNBA.

${ }^{15}$ La carte des blocs pétroliers et gaziers reflète la variété des participants : entreprises privées des pays de l'OCDE (Total, Repsol, Dana, Wintershall), firmes publiques asiatiques (Petronas, CNPC) et sociétés sans expérience pétrolière de diverses origines. 
les capacités de coordination et l'intégration des enjeux environnementaux du multi-usage de l'espace maritime restent limitées.

Ce processus a plusieurs causes : pressions internationales dans le domaine de la pêche ou de la conservation, accumulations diffuses des expériences de projets de coopération, mais aussi conscience croissante des élites politico-administratives de l'intérêt financier des ressources océanes - pour elles-mêmes ou pour le pays. Depuis les années 1970, les milieux économiques nationaux associés à la mer (armateurs, patrons de pêche, industriels transformateurs, intermédiaires) sont étroitement liés aux élites politiques (Blundo, 2007; Choplin et Lombard, 2009), ce qui les amène à peser sur les choix gouvernementaux. À partir du milieu des années 2000, une partie des décisions concernant la gestion des activités maritimes est influencée par l'opposition entre un courant favorable à l'activité pétrolière (ministère du Pétrole, Société mauritanienne des hydrocarbures, ministère des Finances) et une tendance plus prudente quant à ses impacts sur les écosystèmes (ministère de l'Environnement, PNBA, voire ministère de la Pêche). Il n'est pas anodin que les ministères du Pétrole (2005) et de l'Environnement ${ }^{16}$ (2006) aient été créés presque au même moment. L'État mauritanien n'est pas monolithique : ses arbitrages expriment de tels rapports de force changeants. Cependant, la vitesse élevée de rotation des fonctionnaires dans les secteurs tant de la pêche que de l'environnement, reflet de l'instabilité politique et du besoin de maintenir les équilibres ethnico-tribaux (Marchesin, 1992 ; Blundo, 2007), n'est pas favorable à l'adoption et à la mise en œuvre de solutions concertées.

Ainsi, les choix mauritaniens en matière d'adhésion à la législation internationale maritime sont révélateurs de la conscience croissante à la fois des enjeux qui y sont associés et des difficultés à les prendre en charge. Ayant ratifié la convention sur le droit de la mer de 1982, ainsi qu'un grand nombre de conventions et d'accords de l'OMI, en particulier la convention Marpol (Marine Pollution) et la plupart de ses annexes, la Mauritanie dispose d'une base solide pour construire son droit maritime interne - mais cela n'a pas encore été parachevé. Au plan régional, la Mauritanie est membre de la commission sous-régionale des Pêches, mais elle n'a pas ratifié la Convention d'Abidjan relative à la coopération en matière de protection et de mise en valeur du milieu marin et des zones côtières de la région de l'Afrique de l'Ouest et du centre, adoptée en 1981, qui constitue le cadre de

\footnotetext{
${ }^{16}$ Ce dernier a été créé d'abord sous la forme d'un secrétariat d'État, puis d'un ministère délégué auprès du Premier ministre chargé de l'Environnement, avant de devenir ministère à part entière.
}

coopération des vingt-deux pays de la façade atlantique africaine pour l'environnement marin ${ }^{17}$.

Pour des raisons historiques, la Mauritanie est mieux armée pour faire face aux enjeux de pêche qu'à ceux de surveillance et de contrôle de la pollution marine, qu'ils soient liés au trafic maritime ou à l'activité pétrolière offshore. La participation à la commission sousrégionale des Pêches et l'absence d'engagement dans la convention d'Abidjan sont symptomatiques de ses priorités. On peut émettre l'hypothèse que, pour les élites politico-administratives mauritaniennes, la préservation immédiate de la rente halieutique est d'autant plus prioritaire que la sphère environnementale - également pourvoyeuse de rente - semble largement contrôlée par les acteurs de la coopération internationale.

La distribution des moyens matériels et humains d'intervention en mer confirme ce déséquilibre. Alors que le secteur de la pêche semble relativement bien organisé - qu'il s'agisse de suivre l'évolution des stocks, de surveiller les débarquements ou de contrôler la pêche en mer -, le contrôle des autres activités maritimes est balbutiant. De plus, comme dans beaucoup de pays, la création récente d'un ministère de l'Environnement soulève des problèmes d'organisation délicats. C'est ainsi que ce nouveau ministère ne dispose d'aucun moyen pour contrôler les rejets de polluants. Si une pollution de la mer affectait le littoral, la responsabilité de l'intervention relèverait $d$ 'autres ministères, alors que c'est sur celui de l'Environnement que la pression de l'opinion publique s'exercerait. Un plan Polmar (Pollution maritime) a bien été élaboré, mais son approbation est liée à un décret lui-même conditionné par la promulgation du Code de l'environnement maritime (Ruë, 2005), empêchée jusqu'à aujourd'hui par les rivalités entre ministères (Pêche, Transport, Environnement).

\section{Les régulations à construire}

Les différentes formes d'utilisation de l'espace maritime et de ses ressources se sont déployées en se superposant, sans arbitrage sur les contradictions éventuelles pouvant surgir. Les demandes successives de régulation en provenance des activités de la pêche, du pétrole, du transport ou de l'environnement ont été traitées séparément. En l'absence d'une autorité maritime centrale, les négociations ont donné lieu à des accords fragmentés entre des parties prenantes étrangères et des ministères non coordonnés entre eux. Aujourd'hui, la situation organisationnelle et institutionnelle est devenue quasi inextricable. D'autant plus que, comme tout État, l'État mauritanien intervient en situation de pouvoir partagé

\footnotetext{
17 Quatorze pays ont ratifié cette convention que le Programme des Nations unies pour l'environnement (PNUE) s'efforce actuellement de revitaliser.
} 
et doit à chaque instant prendre en compte une grande variété de demandes (Matus, 1987). Comme tous les pays les moins avancés, il doit aussi faire face à des situations de forte asymétrie. La reconnaissance de cet état de fait par une partie de l'élite mauritanienne - formée dans un cadre sectoriel - a conduit à une esquisse de modification des approches de régulation au niveau central de l'État, à partir des débats intenses développés autour des activités pétrolières.

Il semble ainsi que l'on s'achemine vers de nouvelles postures d'interaction entre l'État et les usagers de la mer mauritanienne. À défaut d'une volonté explicite de régulation de l'espace maritime, l'évolution de divers conflits pourrait concourir à l'émergence d'une prise de conscience des enjeux globaux de la gestion de la mer. Des conflits répétés entre pêcheurs artisanaux et industriels ont obligé à des négociations sur de nouvelles règles au sein du secteur de la pêche. Des conflits entre les ONG en appui au PNBA et le secteur de la pêche ont mené à une meilleure prise en compte de la protection du parc lors des négociations sur les accords de pêche avec l'Union européenne. L'arrivée du pétrole et les conflits entre la compagnie Woodside et le gouvernement mauritanien ont permis d'initier un dialogue entre gouvernement, firmes pétrolières et organisations de la société civile. En Mauritanie, les composantes de la société civile les mieux structurées sont issues des organisations de défense de la démocratie et des droits de l'homme. Elles ont notamment contribué activement au débat pétrolier à travers leur participation à la coalition mauritanienne «Publiez ce que vous payez ». Dans le domaine environnemental, en revanche, la société civile nationale est encore balbutiante. Les débats ont été alimentés en grande partie par des ONG internationales (FIBA, UICN, etc.). Ils ont été relayés par la presse, très vivante et relativement indépendante, fort intéressée par les enjeux environnementaux soulevés par l'exploitation pétrolière. La pérennisation de ces débats dépendra notamment de l'évolution de la situation politique nationale. L'amélioration de la gouvernance environnementale en dépend dans une large mesure.

\section{Conclusion}

Comment la complexité croissante des activités maritimes et les risques qui y sont associés sont-ils gérés en Mauritanie?

Les dirigeants mauritaniens regardent aujourd'hui vers la mer plus que par le passé, parce que l'océan fournit désormais l'essentiel de la rente étatique. Celleci provient principalement des prélèvements fiscaux, des accords de pêche, des contrats pétroliers et de l'aide internationale. En mer comme sur terre, la Mauritanie donne l'impression d'un système social et politique organisé à partir d'une base sociopolitique tribale et nomade, commerçante et rentière - qui n'exerce qu'un contrôle territorial tout juste suffisant pour capter les flux nécessaires à la reproduction de ses ressources. Mais cette perception culturaliste ne doit pas être surestimée. Car les relations de l'État à la mer sont caractérisées à la fois par l'intégration des ressources maritimes dans le système rentier clientéliste, le coût élevé du contrôle territorial sur un espace fluide et les effets de la dépendance qui dispensent d'exercer des fonctions de contrôle, d'administration, de gestion des ressources et des risques. Ce contexte de pays " moins avancé », la nature du système politique et ses liens avec l'extérieur semblent déterminer davantage le rapport aux ressources que leur maritimité. Pour le dire autrement, la mer contribue sans grande originalité aux stratégies d'extraversion (Bayart, 1999) qui président à la trajectoire de la société et de l'État mauritaniens.

Si la mer apparaît comme un «confins de la gouvernance » (Van Vliet, 1997) pour les acteurs mauritaniens, on décèle cependant une tension croissante entre la logique basée sur la captation des rentes et une autre forme de contrôle, plus territorial, plus gestionnaire et planificateur, héritage de projets exogènes qui ont influencé un retournement des représentations d'une partie de l'élite. Chemin faisant, des apprentissages ont été accumulés au sein de l'État dans le domaine de la gestion de son espace maritime, même s'ils apparaissent encore le plus souvent fragmentés. L'irruption des questions pétrolières, en révélant les risques latents du multi-usage de l'espace marin, a favorisé des débats nouveaux autour de ces enjeux. Dans un État où le jeu politique repose sur une intrication complexe entre structures sociales tribales, armée et système d'accumulation extraverti, les positionnements politiques récents (pro ou anti-coup d'État de 2008) ne peuvent être lus en termes simples d'opposition entre courant conservateur - favorable à une rente pétrolière destinée à être patrimonialisée - et tendance progressiste - consciente des enjeux de préservation environnementale. En revanche, si l'instabilité des dernières années n'a pas été favorable à la mise en place de règles du jeu claires concernant la gouvernance de l'espace maritime, les débats incessants qui ont caractérisé cette période ont probablement élargi les marges de manœuvre des innovateurs.

La possible montée en puissance de l'exploitation des hydrocarbures place ainsi la Mauritanie face à une bifurcation (Capoccia et Kelemen, 2007; Van Vliet et Magrin, 2009) : l'État peut se dissoudre devant l'afflux des nouvelles ressources - tant extractives qu'issues des trafics parfois illégaux - qui participent aux nouvelles formes d'insertion du Sahara dans l'espace mondialisé. Mais il peut aussi bénéficier des débats qui accompagnent les nouvelles activités pétrolières pour explorer de nouvelles voies et se renforcer tout en consolidant le processus de démocratisation. 


\section{Références}

Antil, A., 1999. Le Territoire d'État en Mauritanie : genèse, héritage, représentation. Thèse de doctorat en géographie, Université de Rouen.

Augé, B., 2007. Les Enjeux géopolitiques du pétrole en République islamique de Mauritanie. Mémoire de master 2, Université Paris 8, Saint-Denis.

Bayart, J.-F., 1999. L'Afrique dans le monde : une histoire d'extraversion, Critique internationale, 5, 97-120.

Blundo, G., 2007. Graisser la barbe : mécanismes et logiques de la corruption en Mauritanie. Note de synthèse établie à l'intention de la Délégation de la Commission européenne en Mauritanie, Bruxelles.

Bonte, P., 2000. Faire fortune au Sahara : permanences et ruptures, Autrepart, 16, 49-65.

Bonte, P., 2001. La Montagne de fer : la Snim, Paris, Karthala.

Boulay, S., 2008. Mutations techniques, changements sociaux survenus chez les pêcheurs imrâgen, des années 1970 à nos jours. Rapport PNBA-Adage, Nouakchott.

Campredon, P., 2000. Entre le Sahara et l'Atlantique : le Parc national du Banc d'Arguin, Arles, FIBA.

Capoccia, G., Kelemen, R.D., 2007. The study of critical junctures: Theory, narrative and counterfactuals in historical institutionalism, World Politics, 59, 3, 341-369.

Choplin, A., 2006. Fabriquer des villes-capitales entre monde arabe et Afrique noire: Nouakchott (Mauritanie) et Khartoum (Soudan), étude comparée. Thèse de doctorat en géographie, Université Paris 1 Panthéon-Sorbonne, Paris.

Choplin, A., Lombard, J., 2009. La « Mauritanie off shore». Extraversion économique, État et sphères dirigeantes, Politique africaine, 114, 85-102.

Ferguson, J., 2006. Global Shadows: Africa in the Neoliberal World Order, Durham and London, Duke University Press.

Goodland, R., 2006. Mauritania: Oil and Gas Sector Environment Mission Report, GTZ, Nouakchott.

Humphreys, M., Sachs, J.D., Stiglitz, J.E. (Eds), 2007. Escaping the Resource Curse, New York, Columbia University Press.

Kloff, S., Van Spanje, T., 2004. A Review of Woodside's Draft Environmental Impact Statement of the Chingueti's Offshore Oil Development Project in Mauritania, Mineral Policy Institute, [s.l.], Australia.

Kloff, S., Wicks, C., 2005. Gestion environnementale de l'exploitation de pétrole offshore et du transport maritime pétrolier, [Nouakchott], FIBA/WWF/IUCN/PRCM.

Le Cœur, C., 1994. La Pêche en Mauritanie : du désert à la mer ou l'appropriation d'un espace halieutique. Thèse de géographie, Université Montpellier 3, Montpellier.

Lechartier, C., 2005. L'Espace nomade du pouvoir politique en Mauritanie : des lieux de la bediya de l'Est à la capitale. Thèse de doctorat en géographie, Université de Rouen.

Magrin, G., 2010. Rentes, territoires et développement. Que tout change pour que rien ne change?, in Pourtier, R. (Ed.), 1960$2010: 50$ ans d'indépendances africaines, BAGF, 2010-1, 56-68.

Magrin, G., Van Vliet, G., 2009. The use of oil revenues in Africa, in Lesourne, J. (Ed.), Governance of Oil in Africa: Unfinished Business, Paris, IFRI, 103-163.

Marchesin, P., 1992. Tribus, ethnies et pouvoir en Mauritanie, Paris, Karthala.

Reçu le 20 janvier 2009. Accepté le $1^{\text {er }}$ décembre 2010.
Marfaing, L., 2005. Du savoir-faire sénégalais en matière de pêche sur les côtes mauritaniennes : une approche historique, Stichproben : Wiener Zeitschrift für kritische Afrikastudien, 8, 69-98.

Matus, C., 1987. Adios, señor Presidente: planificación, antiplanificación y gobierno, Caracas, Pomaire.

Ministère de la Culture, de la Jeunesse et des Sports (Ed.), 2006. Nouakchott, capitale de la Mauritanie : 50 ans de défi, SaintMaur-des-Fossés, Sépia.

Oilwatch, 2005. Exploitation pétrolière en Mauritanie, [s.1.] (http://www.oilwatch.org/2005/francais/documentos/ap_ mauritania_fra.pdf).

Ould Ahmed Salem, Z., 2008. Le Partenariat Union européenneAfrique dans l'impasse? Le cas des accords de pêche. ASC [African Studies Centre] Working Paper 78/2008, Philadelphie.

Ould Cheikh, A.W., 2002. L'Identité imraguen et la gestion locale de l'aire du PNBA : approche historique et sociologique, Nouakchott, Parc national du banc d'Arguin.

Retaillé, D., 2006. Espace mobile, territorialité sans territoire et urbanité sans ville, in Retaillé, D. (Ed.), La Ville ou l'État? Développement politique et urbanité dans les espaces nomades ou mobiles (Mauritanie, Sénégal, Inde et retour), Mont-SaintAignan, Publications des Universités de Rouen et du Havre.

Rosser, A., 2006. The Political Economy of the Resource Curse: A Literature Survey. IDS Working Paper 268, Centre for the Future State, Brighton.

Ruë, O., 2005. Éléments d'analyse pour l'établissement d'une position du PNBA par rapport au développement de l'activité pétrolière off-shore. Note du PNBA, Nouakchott.

Semelin, J., 2005. Synthèse bibliographique à propos des impacts écologiques et des aspects réglementaires de l'exploitation pétrolière en mer, [Arles], FIBA/PRCM.

Soares de Oliveira, R., 2007. Oil and Politics in the Gulf of Guinea, London, Hurst \& Company.

Toupet, C., 1968. Les activités maritimes de Port Étienne, Cahiers d'Outre-Mer, XXI, 84, 1-18.

Van Vliet, G., 2002. Rapport final de la mission d'appui au développement organisationnel du Parc national du Banc d'Arguin (PNBA), 26 février au 15 mars.

Van Vliet, G., 1997. Le Pilotage aux confins mouvants de la gouvernance : économie, politique, écologie et régulation en Amazonie colombienne, 1975-1990. Thèse de doctorat en sciences économiques, Université Paris 1 Panthéon-Sorbonne, Paris (http://tel.ccsd.cnrs.fr/documents/archives0/00/00/74/90/ index_fr.html).

Van Vliet, G., Magrin, G., 2009. Public steering in the hydrofuel sector. Conditions for trajectory bifurcation in Chad and Mauritania, 21st World Congress of Political Science, IPSA, July 12-16, Santiago (Chile).

Van Vliet, G., Magrin, G., Van Dessel, B., Chabason, L., 2009. Panel scientifique indépendant sur les activités pétrolières et gazières en République islamique de Mauritanie. Rapport définitif, UICN, Nouakchott.

Walther, O., Retaillé, D., 2008. Le modèle sahélien de la circulation, de la mobilité et de l'incertitude spatiale, in Michel, L. (Ed.), Variations et dynamiques urbaines, Autrepart, 47, 109-124.

Woodside, 2003. Chinguetti Development Project. Draft Environmental Impact Statement, Nouakchott.

Woodside, 2005. Chinguetti Development Project. Environmental Impact Statement, version finale, Nouakchott. 